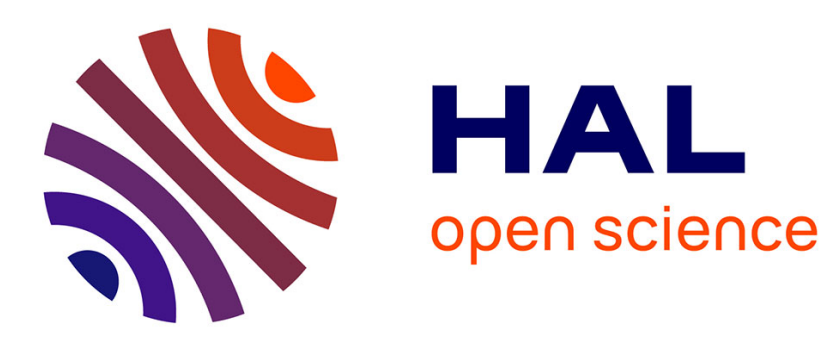

\title{
Nucleation and interfacial adsorption in ternary systems
} Thomas Philippe

\section{To cite this version:}

Thomas Philippe. Nucleation and interfacial adsorption in ternary systems. Journal of Chemical Physics, 2015, 142 (9), pp.094501. 10.1063/1.4913592 . hal-03035910

\section{HAL Id: hal-03035910 https://hal.science/hal-03035910}

Submitted on 2 Dec 2020

HAL is a multi-disciplinary open access archive for the deposit and dissemination of scientific research documents, whether they are published or not. The documents may come from teaching and research institutions in France or abroad, or from public or private research centers.
L'archive ouverte pluridisciplinaire HAL, est destinée au dépôt et à la diffusion de documents scientifiques de niveau recherche, publiés ou non, émanant des établissements d'enseignement et de recherche français ou étrangers, des laboratoires publics ou privés. 


\section{A|P| $\begin{aligned} & \text { The Journal of } \\ & \text { Chemical Physics }\end{aligned}$}

\section{Nucleation and interfacial adsorption in ternary systems}

T. Philippe

Citation: The Journal of Chemical Physics 142, 094501 (2015); doi: 10.1063/1.4913592

View online: http://dx.doi.org/10.1063/1.4913592

View Table of Contents: http://scitation.aip.org/content/aip/journal/jcp/142/9?ver=pdfcov

Published by the AIP Publishing

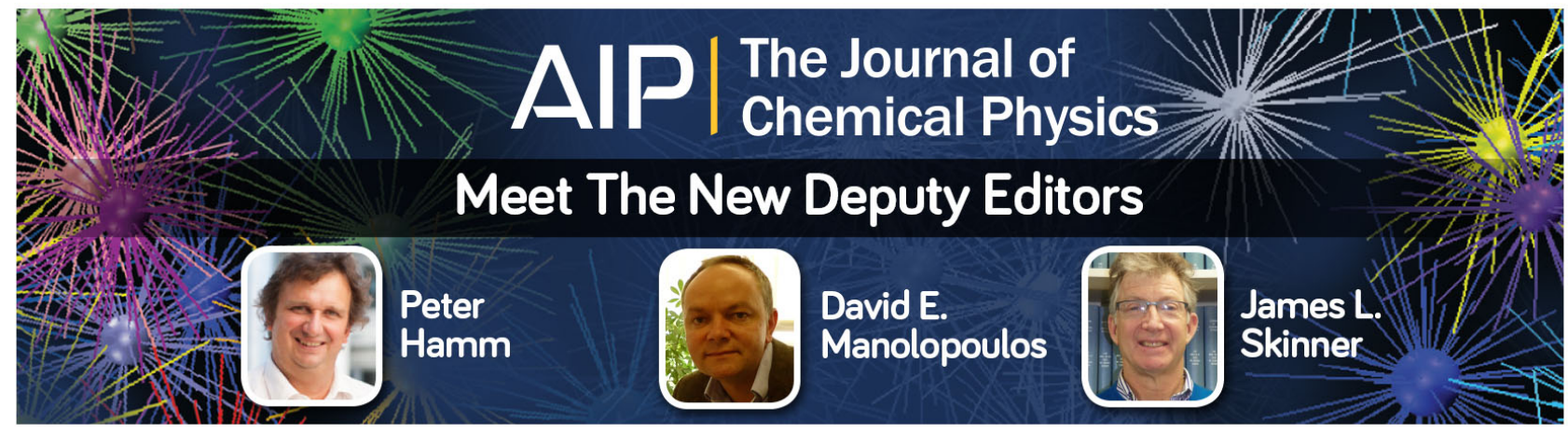




\title{
Nucleation and interfacial adsorption in ternary systems
}

\author{
T. Philippe ${ }^{\text {a) }}$ \\ Normandie Université, Groupe de Physique des Matériaux (GPM), UMR CNRS 6634 BP 12, \\ Avenue de l'Université, 76801 Saint Etienne du Rouvray, France
}

(Received 17 October 2014; accepted 16 February 2015; published online 4 March 2015)

\begin{abstract}
Nucleation is studied in incompressible ternary fluids by examining the topology of the overall landscape of the energy surface. Minimum free energy paths for nucleation (MFEPs) of a single nucleus in an infinite matrix are computed with the string method in the framework of the continuum theory of nucleation for the regular solution. Properties of the critical nucleus are compared with the predictions of the classical nucleation theory. MFEPs are found to exhibit complex nucleation pathways with non-monotonic variations of compositions in the interfacial region, specifically adsorption of a component. In the symmetric regular solution, the minority component is found to segregate at the interface during nucleation with a concomitant depletion of the nucleus core, resulting in unpredicted partition of the non-selective component. Despite increasing the gradient energy, such inhomogeneity in composition is shown to lower the nucleation barrier. () 2015 AIP Publishing LLC. [http://dx.doi.org/10.1063/1.4913592]
\end{abstract}

\section{INTRODUCTION}

Numerous first-order phase transitions proceed via nucleation and growth of a new phase. The thermodynamics of such a process was first investigated by Gibbs ${ }^{1}$ and van der Waals. ${ }^{2,3}$ The initial formulation of the nucleation-growth model was performed by Gibbs ${ }^{4-9}$ and is known as the classical theory of nucleation. Many developments and refinements have been brought to the early theories of pioneers such as Becker, ${ }^{10}$ Zeldovich, ${ }^{11}$ and Turnbull ${ }^{12}$ who included latent nucleation time and regression effects. Cahn and Hilliard $(\mathrm{CH})^{13,14}$ reexpressed the van der Waal's method to develop a continuum theory of nucleation in a two-component incompressible fluid accounting for interfaces diffusivity. It was shown that classical nucleation theory (CNT) is only valid for low supersaturation. ${ }^{14}$ Since its introduction, the question under which conditions the conventional theory of nucleation is accurate has been debated and this debate continues till today. ${ }^{15}$

Nuclei properties can differ from those of bulk phases and are expected to result from a complex interplay between kinetics and thermodynamics in a multicomponent system. Nucleation of critical droplets is a rare phenomenon and its study requires an accurate description, at least, of the thermodynamics driving the process. This is not trivial, even if only nucleation of a single droplet in an infinite matrix is considered. The reason is that the exhaustive study of the free energy surface is generally impossible because of the very high degrees of freedom. In nucleation, the minimum free-energy pathway (MFEP) is the following: the subcritical nucleus ascends the energy landscape until the saddle point (the critical nucleus) and the supercritical nucleus descends along the valley and enters in the growth stage. MFEPs are the most probable routes even if real paths might wander around MFEPs through a

\footnotetext{
a) Author to whom correspondence should be addressed. Electronic mail: thomas.philippe1984@gmail.com
}

thermal fluctuation or kinetics effects. MFEPs can therefore be viewed as the transition paths of maximum likelihood, ${ }^{16,17}$ as shown in the context of large deviation theory. ${ }^{18}$ Indeed, the Freidlin-Wentzell theory of large deviations predicts that rare events happen almost always in the same predictable way because any other way is even much more unlikely. Precisely, the reactive trajectories follow the MFEP when the temperature $(k T)$ is much smaller than the energy barrier. The flux carried by the probability current of the reactive trajectories is concentrated in a small tube around the MFEP. Dynamics of the transformation proceeds by long waiting periods around the metastable states followed by sudden jumps from one state to another. These jumps define, in terms of trajectories, a small tube around the MFEP. In nucleation, the MFEP represents the mean behavior averaged over a large amount of clusters and only a small amount of nuclei have a behavior very different than the average.

As the configuration space is multidimensional, some authors have reduced the dimension of the problem by considering that nuclei are chemically homogeneous and have abrupt interfaces. Nucleation was studied in the $(c, R)$ space, where $c$ and $R$ are, respectively, the composition and the radius of the nucleus, ${ }^{19-21}$ by determining the saddle point and the minimum free energy paths. ${ }^{21,22}$ Weakleim and Reiss proposed a molecular theory of nucleation: the $(N, \lambda)$ cluster model where a fixed number of molecules $N$ are confined within a container of radius $\lambda .^{23}$ The projection of the free energy surface onto the $(N, \lambda)$ space was assumed to capture the essential features of the real free-energy surface in multidimensional space. The work of formation of a critical cluster was found to vanish near the spinodal line where the critical size diverges, similarly to the $\mathrm{CH}$ theory. ${ }^{14}$ Trinkaus extended the Fokker-Planck equation to multiple dimensional spaces. ${ }^{24}$

Saddle point of the free energy is commonly admitted to thermodynamically set the properties of the critical nuclei. However, as capillarity is neglected in the $(c, R)$ or $(N, \lambda)$ 
theories, change in chemical potential due to interface curvature is not considered and local equilibrium at the interface does not hold. This is not the case with classical nucleation theory as the Zeldovich equation for classical nucleation and the growth equation combined with the Ostwald-Freundlich boundary condition (capillarity) lead to the same critical radius, as recently remarked by Iwamatsu. $^{25}$ It is well known, for example, that the critical radius for coarsening coincides with the critical radius for nucleation. ${ }^{26}$ Moreover, theories with abrupt interfaces fail to properly describe nucleation for relatively high supersaturation, when nuclei become particularly diffuse. Thus, an appropriate description of nucleation requires coincidence between thermodynamic and kinetic critical clusters and needs to account for the diffusive nature of the interfaces such as the $\mathrm{CH}$ theory.

Recent progresses in computational science ${ }^{27-30}$ allow for the investigation of nucleation in multidimensional spaces, providing a better understanding of nucleation phenomena. Iwamatsu studied nucleation using the numerically efficient cell-dynamics method coupled to a phase field model. ${ }^{31}$ Lutsko studied the liquid-vapor nucleation in the modified-core van der Waals model density functional theory ${ }^{32,33}$ using the nudged elastic-band method. ${ }^{27,28}$ Wood and Wang used the self-consistent field theory for a binary polymer mixture and have investigated nucleation pathways. ${ }^{34}$

Recently, a numerical technique, the string method, has been developed to determine the MFEP connecting two metastable states in multidimensional configuration spaces. ${ }^{29,30}$ This method has been successfully applied to the study of membrane adhesion ${ }^{35}$ and capillary condensation ${ }^{36}$ and to investigate nucleation of ordered phases in block copolymers in the framework of the self-consistent field theory. ${ }^{37}$ Voigt et al. have used the string method to investigate crystallization from the liquid with the phase field crystal model. ${ }^{38}$ Nucleation in solids has been studied using the phase field equation and the string method with a focus on the morphology of critical and equilibrium nuclei. ${ }^{39,40}$ The string method also allowed for the investigation of nucleation process in a mean field theory ${ }^{41}$ and in the $\mathrm{CH}$ theory. ${ }^{42}$ The string method was also applied, in other contexts, to the study of nucleation process in the $\mathrm{CH}$ model. ${ }^{43,44}$

Philippe and Blavette ${ }^{42}$ have used the string method to study nucleation in binary systems. In this work, nucleation in a two-component incompressible system was studied by examining the topology of the free-energy landscape. The properties of a single cluster during nucleation were derived from the MFEP. In particular, a detailed description of the nucleation process was provided for a regular solution for increasing supersaturation up to the spinodal line. The composition of the critical nucleus was found, near the spinodal line, to significantly differ from its bulk value. The size of the critical nucleus was found to diverge both near the binodal and spinodal lines and the work required to form a critical nucleus was found to vanish near the spinodal line, describing a smooth transition between nucleation and the spinodal regime. In terms of the physics of nucleation, a universal two-step behavior was found: nuclei sharply enrich up to equilibrium and then grow with a progressive enrichment of the nucleus composition until maximizing the driving force for nucleation.
In the present work, MFEPs are computed with no constraint on mass conservation, i.e., the small amount of solute atoms (or molecules) incorporated into the growing nucleus is negligible and does not affect the far-field matrix composition. In this study, we are particularly interested in the properties of the critical nucleus for ternary systems. However, we shall mention that growth of post-critical clusters requires diffusion and, as a consequence, depletes (or enriches) the region surrounding the particles. Thus, rapidly after reaching its critical size the nucleus is expected to satisfy the OstwaldFreundlich condition.

Here, MFEPs for nucleation are computed for ternary regular solutions in the framework of the $\mathrm{CH}$ theory. Results are compared to classical nucleation theory. The purpose is to understand nucleation in ternary systems. Section II presents the $\mathrm{CH}$ free energy and the regular solution model in multicomponent systems, and Sec. III describes the methods. Section IV presents and discusses the numerical results.

\section{THE GOVERNING EQUATIONS}

\section{A. The free energy}

Due to matter conservation, an important sum rule on the mole (or atomic) fraction of each species exists,

$$
\sum_{i=1}^{N} c_{i}=1
$$

where $c_{i}$ is the mole fraction of specie $\mathrm{i}$ and $\mathrm{N}$ the total number of components. Following $\mathrm{CH},{ }^{13}$ the bulk Helmholtz free energy, $F$, of a non-uniform solution is [Refs. 45-47, see Appendix A]:

$F=\int_{V}\left\{f_{0}\left(c_{1}, c_{2}, \ldots, c_{N-1}\right)+\sum_{i=1}^{N-1} \sum_{j=1}^{N-1} \kappa_{i j} \nabla c_{i} \nabla c_{j}\right\} d V$,

where the $\kappa_{i j}$ are the gradient energy coefficients and $f_{0}$ is the free energy of the homogeneous solution.

\section{B. The work of formation of a critical nucleus}

At the saddle point of the free-energy surface, the system is in an unstable equilibrium; it is here that a fluctuation in composition becomes a critical nucleus. It is also where the free energy must be stationary with respect to a change in composition. Thus, the critical nucleus is defined such that the integral in Eq. (2) is stationary subject to the condition that the average composition remains constant, or in a multicomponent system subject to the $\mathrm{N}-1$ constraints,

$$
\int_{V}\left(c_{i}-c_{i}^{0}\right) d V=0 \quad(i=1, N-1),
$$

where $c_{i}^{0}$ are both the initial and average compositions. The work, $W$, required to form a critical nucleus is given by

$$
W=\int_{V}\left\{f_{0}-f_{0}^{0}+\sum_{i=1}^{N-1} \sum_{j=1}^{N-1} \kappa_{i j} \nabla c_{i} \nabla c_{j}\right\} d V,
$$

where $f_{0}^{0}$ is the free energy of the initial metastable solution. The driving force for nucleation $\Delta f_{0}$ is given by 


$$
\Delta f_{0}=f_{0}-f_{0}^{0}-\left.\sum_{i=1}^{N-1}\left(c_{i}-c_{i}^{0}\right) \frac{\partial f_{0}}{\partial c_{i}}\right|_{c_{1}=c_{1}^{0}, \ldots, c_{N-1}=c_{N-1}^{0}} .
$$

For an isotropic system, the critical nucleus is spherically symmetric. Using Eqs. (3) and (5), the work, $W$, to form a critical nucleus can be written as

$$
W=4 \pi \int_{0}^{\infty}\left\{\Delta f_{0}+\sum_{i=1}^{N-1} \sum_{j=1}^{N-1} \kappa_{i j} \frac{d c_{i}}{d r} \frac{d c_{j}}{d r}\right\} r^{2} d r .
$$

Applying the Euler equation to Eq. (6) yields as the requirement for an extrema in the free energy,

$$
\begin{gathered}
2 \sum_{j=1}^{N-1} \kappa_{1 j} \frac{d^{2} c_{j}}{d r^{2}}+\frac{4}{r} \sum_{j=1}^{N-1} \kappa_{1 j} \frac{d c_{j}}{d r}=\frac{\partial \Delta f_{0}}{\partial c_{1}}+\lambda_{1}, \\
2 \sum_{j=1}^{N-1} \kappa_{2 j} \frac{d^{2} c_{j}}{d r^{2}}+\frac{4}{r} \sum_{j=1}^{N-1} \kappa_{2 j} \frac{d c_{j}}{d r}=\frac{\partial \Delta f_{0}}{\partial c_{2}}+\lambda_{2}, \\
\vdots \\
2 \sum_{j=1}^{N-1} \kappa_{N-1, j} \frac{d^{2} c_{j}}{d r^{2}}+\frac{4}{r} \sum_{j=1}^{N-1} \kappa_{N-1, j} \frac{d c_{j}}{d r}=\frac{\partial \Delta f_{0}}{\partial c_{N-1}}+\lambda_{N-1},
\end{gathered}
$$

where $\lambda_{i}$ are Lagrange multipliers introduced by the subsidiary condition of Eq. (3). For a sufficiently large system, the compositions remain unchanged far from the critical nucleus and there are no composition gradients, thus

$$
\left.\frac{\partial \Delta f_{0}}{\partial c_{i}}\right|_{c_{1}=c_{1}^{0}, \ldots, c_{N-1}=c_{N-1}^{0}}+\lambda_{i}=0 \quad(i=1, N-1),
$$

which gives $\lambda_{i}=0$ for all $i=1, N-1$.

The Euler-Lagrange equation can be written in a more compact form, ${ }^{45}$

$$
2 \kappa \frac{d^{2} \mathbf{c}}{d \mathbf{r}^{2}}+\frac{4}{r} \kappa \frac{\mathbf{d c}}{\mathbf{d r}}=\frac{\partial \Delta f_{0}}{\partial \mathbf{c}}
$$

subject to the following boundary conditions:

$$
\frac{d c_{i}}{d r}=0 \quad \text { for } r \rightarrow 0 \quad \text { and } \quad r \rightarrow \infty \quad(i=1, N-1)
$$

and

$$
c_{i}=c_{i}^{0} \quad \text { for } r \rightarrow \infty \quad(i=1, N-1) .
$$

The partial derivatives of the free energy $F$ with respect to compositions intervene in the $\mathrm{CH}$ equation,

$$
\frac{d c_{i}}{d t}=\sum_{j=1}^{N-1} M_{i j} \nabla^{2} \tilde{\mu}_{j}
$$

where $\mathbf{M}$ is the mobility matrix and $\tilde{\mu}_{j}$ the generalized chemical potential of specie $j$,

$\tilde{\mu}_{j}=\frac{\delta F}{\delta c_{j}}=\frac{\partial f_{0}}{\partial c_{j}}-2 \sum_{i=1}^{N-1} \kappa_{j i} \frac{d^{2} c_{i}}{d r^{2}}-\frac{4}{r} \sum_{i=1}^{N-1} \kappa_{j i} \frac{d c_{i}}{d r}$.

Using Eqs. (5) and (7) in Eq. (13) gives

$$
\tilde{\mu}_{j}=\left.\frac{\partial f_{0}}{\partial c_{j}}\right|_{c_{1}=c_{1}^{0}, \ldots, c_{N-1}=c_{N-1}^{0}} \quad(j=1, N-1) .
$$

The above equation implies that the generalized chemical potentials are constant along the radial composition profile of the critical nucleus; this makes the critical nucleus also unstable kinetically, i.e., $d c_{i} / d t=0$, since infinitesimal composition fluctuations will make the cluster shrink or grow. Thus, the critical cluster for nucleation coincides with the critical cluster for growth.

Kinetics effects, such as presence of a slow component, are expected to affect the composition profiles of sub- and supercritical nucleus evolving by diffusion with the $\mathrm{CH}$ equation. However, in a sufficiently large system, typically much larger than the diffusion distances, such effects have no influence on the radial composition profile of the critical nucleus that, in any case, must satisfy Eq. (8), i.e., changes in compositions far from the nucleus are negligible, and therefore Eq. (9).

\section{The interfacial energy}

Following $\mathrm{CH}$, the interfacial free energy (by unit area), $\sigma$, is given by Ref. 45 ,

$$
\begin{aligned}
\sigma= & \int_{-\infty}^{+\infty}\left\{f_{0}\left(c_{1}, c_{2}, \ldots, c_{N-1}\right)\right. \\
& +\sum_{i=1}^{N-1} \sum_{j=1}^{N-1} \kappa_{i j} \nabla c_{i} \nabla c_{j}-c_{1} \bar{\mu}_{1}-c_{2} \bar{\mu}_{2} \\
& \left.-\cdots-\left(1-\sum_{i=1}^{N-1} c_{i}\right) \bar{\mu}_{N}\right\} d x,
\end{aligned}
$$

where the $\bar{\mu}_{j}$ are the chemical potentials at equilibrium.

Using

$$
\begin{aligned}
\Delta \bar{f}_{0} & =f_{0}-\bar{f}_{0}-\left.\sum_{i=1}^{N-1}\left(c_{i}-\bar{c}_{i}\right) \frac{\partial f_{0}}{\partial c_{i}}\right|_{c_{1}=\bar{c}_{1}, \ldots, c_{N-1}=\bar{c}_{N-1}} \\
& =f_{0}-\bar{f}_{0}-\sum_{i=1}^{N-1}\left(c_{i}-\bar{c}_{i}\right)\left(\bar{\mu}_{i}-\bar{\mu}_{N}\right),
\end{aligned}
$$

with $\bar{f}_{0}=\sum_{i=1}^{N} \bar{c}_{i} \bar{\mu}_{i}$, the free energy density at the equilibrium compositions $\bar{c}_{i}$, the interfacial free energy can be written as

$$
\sigma=\int_{-\infty}^{+\infty}\left\{\Delta \bar{f}_{0}+\sum_{i=1}^{N-1} \sum_{j=1}^{N-1} \kappa_{i j} \nabla c_{i} \nabla c_{j}\right\} d x .
$$

The $\mathrm{N}-1$ composition profiles across a flat interface are defined such that the integral in Eq. (17) is stationary. Application of the Euler equation gives

$$
\begin{array}{r}
2 \sum_{j=1}^{N-1} \kappa_{1 j} \nabla^{2} c_{j}=\frac{\partial \Delta \bar{f}_{0}}{\partial c_{1}}, \\
2 \sum_{j=1}^{N-1} \kappa_{2 j} \nabla^{2} c_{j}=\frac{\partial \Delta \bar{f}_{0}}{\partial c_{2}},
\end{array}
$$

$$
2 \sum_{j=1}^{N-1} \kappa_{N-1, j} \nabla^{2} c_{j}=\frac{\partial \Delta \bar{f}_{0}}{\partial c_{N-1}}
$$


or in matrix notation,

$$
2 \kappa \nabla^{2} \mathbf{c}=\frac{\partial \Delta \bar{f}_{0}}{\partial \mathbf{c}}
$$

Note that Eq. (9), for a spherical interface, is equivalent to the above equation as $(4 \mathbf{\kappa} / r) \mathbf{d c} / \mathbf{d r} \rightarrow \mathbf{0}$ and $c_{i}^{0} \rightarrow \bar{c}_{i}$ at equilibrium.

From Eq. (18), the following relation for the composition profile of a flat interface holds

$$
\Delta \bar{f}_{0}=\sum_{i=1}^{N-1} \sum_{j=1}^{N-1} \kappa_{i j} \nabla c_{i} \nabla c_{j}
$$

and we find

$$
\sigma=2 \int_{-\infty}^{+\infty} \Delta \bar{f}_{0} d x
$$

that is equivalent to the formulation of the interfacial energy in the binary case. ${ }^{13}$ In the above equation, $\Delta \bar{f}_{0}$ represents the distance between the equilibrium hyperplane and the $\mathrm{N}$ dimensional free energy surface. ${ }^{13,45}$

\section{The regular solution}

At this point, we consider a simple regular solution. The free energy, $f_{0}$, of such a solution is given by

$$
\frac{f_{0}}{N_{v} k T}=\sum_{i=1}^{N-1} \sum_{j=1, j>i}^{N} \frac{2}{\eta^{i j}} c_{i} c_{j}+\sum_{i=1}^{N} c_{i} \ln c_{i},
$$

with $T$, the temperature, $k$, the Boltzmann constant, $N_{v}$, the number of atoms by unit volume, and the reduced temperatures

$$
\eta^{i j}=\frac{T}{T_{c}^{i j}},
$$

where the $T_{c}^{i j}$ are the critical temperatures. The free energy is expressed as a function of $\mathrm{N}-1$ independent compositions. In order to properly study nucleation in a regular solution, a physical description of the gradient energy coefficients is required, which will be used in the numerical section. We extend the procedure of $\mathrm{CH}^{13}$ and Hoyt ${ }^{45}$ to express the gradient energy coefficients, see Appendix B, in a multicomponent regular solution

$$
\kappa_{i j}=\delta_{i j} N_{v} k T_{c}^{i j} \lambda_{i j}^{2},
$$

where $\lambda_{i j}$ are effective interaction distances, $\delta_{i j}$ is the Kronecker symbol. Equation (24) agrees with the $\mathrm{CH}$ result ${ }^{13}$ in the binary limit and with Hoyt ${ }^{45}$ in ternary systems. We use, in the following, the same approximation employed by Hoyt: ${ }^{45}$ $\lambda_{i j}=\lambda$.

Applying the above result in Eq. (6) gives the work associated to the formation of a critical nucleus in a multicomponent regular solution

$\bar{W}=\frac{W}{4 \pi N_{v} k T \lambda^{3}}=\int_{0}^{\infty}\left\{\Delta f_{0}^{\prime}+\sum_{i=1}^{N-1} \frac{1}{\eta_{i N}}\left(\frac{d c_{i}}{d \bar{r}}\right)^{2}\right\} \bar{r}^{2} d \bar{r}$,

where $\bar{r}=r / \lambda$ and $f_{0}^{\prime}=f_{0} / N_{v} k T$.

Similarly, the free energy of a flat interface can be written as

$$
\bar{\sigma}=\frac{\sigma}{N_{v} k T \lambda}=2 \int_{-\infty}^{\infty} \Delta \bar{f}_{0}^{\prime} d \bar{x}
$$

where $\bar{x}=x / \lambda$.

\section{FRAMEWORK AND METHODS}

It is proposed to study nucleation in two regular solutions, the first system is asymmetric (system 1) and the second is symmetric (system 2). In both cases, the reduced temperatures for the ternary regular solutions have to be specified. For both systems, nucleation is investigated from the binodal line to the spinodal line in varying supersaturation along a defined tieline.

\section{A. System 1}

For studying the asymmetric regular solution, we use $\eta_{13}=2 / 3, \eta_{23}=8 / 9$, and a negative order energy between component 1 and 2, i.e., $\eta_{12}=-8 / 3$, so that to have no miscibility gap in the 1-2 binary solution. The isothermal section of the phase diagram is shown in Fig. 1. The equilibrium state of the system was determined by equating the chemical potential of each component in the possible coexisting phases $m, m=\alpha, \beta, \gamma$, including the mass conservation laws

$$
\begin{gathered}
\mu_{i}^{\alpha}=\mu_{i}^{\beta}\left(=\mu_{i}^{\gamma}\right) \quad i=1,2,3, \\
c_{i}^{0}=\phi^{\alpha} c_{i}^{\alpha}+\phi^{\beta} c_{i}^{\beta}\left(+\phi^{\gamma} c_{i}^{\gamma}\right) \quad i=1,2,3,
\end{gathered}
$$

where $\phi$ is the equilibrium volume fraction of the denoted phase. The above system was solved using the NewtonRaphson method. The phase diagram shows two single-phase regions (denoted $\alpha$ and $\beta$ ) at this temperature and a two-phase region $(\alpha+\beta)$. The dashed line in Fig. 1 is the intersection of the spinodal surface. The spinodal curves are determined by

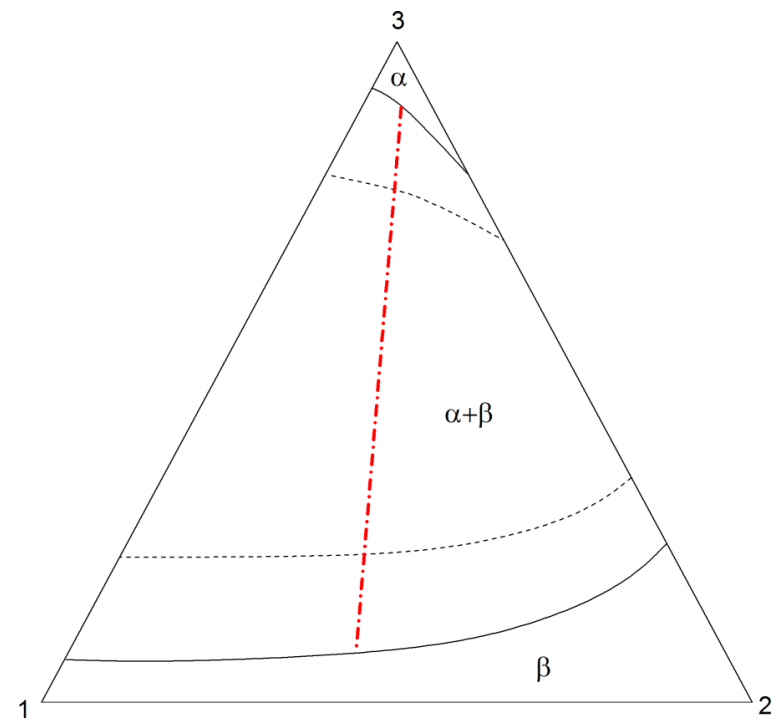

FIG. 1. Phase diagram for the asymmetric ternary regular solution with $\eta_{13}$ $=2 / 3, \eta_{23}=8 / 9$, and $\eta_{12}=-8 / 3$. System 1 . The phase diagram shows two single-phase regions (denoted $\alpha$ and $\beta$ ) at this temperature and a two-phase region $(\alpha+\beta)$. The dashed line is the intersection of the spinodal surface. For the tie-line shown (red line), the equilibrium compositions are $\bar{c}_{1}^{\alpha}=0.039$ and $\bar{c}_{2}^{\alpha}=0.051$ in the $\alpha$ phase and $\bar{c}_{1}^{\beta}=0.514$ and $\bar{c}_{2}^{\beta}=0.401 \mathrm{in} \beta$. The spinodal limit is located at $c_{2}^{s}=0.108\left(c_{1}^{s}=0.116\right)$. 


$$
\left|\begin{array}{cc}
\frac{\partial^{2} f_{0}}{\partial c_{1}^{2}} & \frac{\partial^{2} f_{0}}{\partial c_{2} \partial c_{1}} \\
\frac{\partial^{2} f_{0}}{\partial c_{2} \partial c_{1}} & \frac{\partial^{2} f_{0}}{\partial c_{2}^{2}}
\end{array}\right|=0 .
$$

The tie-line along which nucleation of the $\beta$ phase is investigated is also shown in Fig. 1. In the following, supersaturations are given as a function of the mole fraction of specie 2 only $\left(c_{2}^{0}\right)$, the other compositions being set by the considered tie-line. For the tie-line shown in Fig. 1, the equilibrium compositions are $\bar{c}_{1}^{\alpha}=0.039$ and $\bar{c}_{2}^{\alpha}=0.051$ in the $\alpha$ phase and $\bar{c}_{1}^{\beta}=0.514$ and $\bar{c}_{2}^{\beta}=0.401$ in $\beta$. The spinodal limit is located at $c_{2}^{s}=0.108$ $\left(c_{1}^{s}=0.116\right)$. At equilibrium, the matrix is rich in component 3 , and this component is minority in the $\beta$ phase.

\section{B. System 2}

In the symmetric regular solution, interaction energy between components is equal. The reduced temperatures are set to $2 / 3\left(\eta=\eta_{13}=\eta_{23}=\eta_{12}=2 / 3\right)$. The isothermal section of the phase diagram is shown in Fig. 2, which also shows the specific tie-line along which nucleation, in the two-phase $\alpha+\beta$ region, is investigated. Once again when referring to supersaturation, only one composition $\left(c_{2}^{0}\right)$ will be specified, others are set by the tie-line. The equilibrium compositions are $\bar{c}_{1}^{\alpha}$ $=0.868$ and $\bar{c}_{2}^{\alpha}=0.082$ in $\alpha$ and $\bar{c}_{1}^{\beta}=0.082$ and $\bar{c}_{2}^{\beta}=0.868$ in $\beta$. The spinodal limit is located at $c_{2}^{s}=0.208\left(c_{1}^{s}=0.742\right)$. The third component is minority and non-selective $\left(\bar{c}_{3}^{\alpha}=\bar{c}_{3}^{\beta}\right.$ $=0.05)$, i.e., no partition.

\section{The string method}

Minimum free energy paths are computed using the string method (see Appendix C for more details on the method). Strings are smooth curves with intrinsic parameterization and

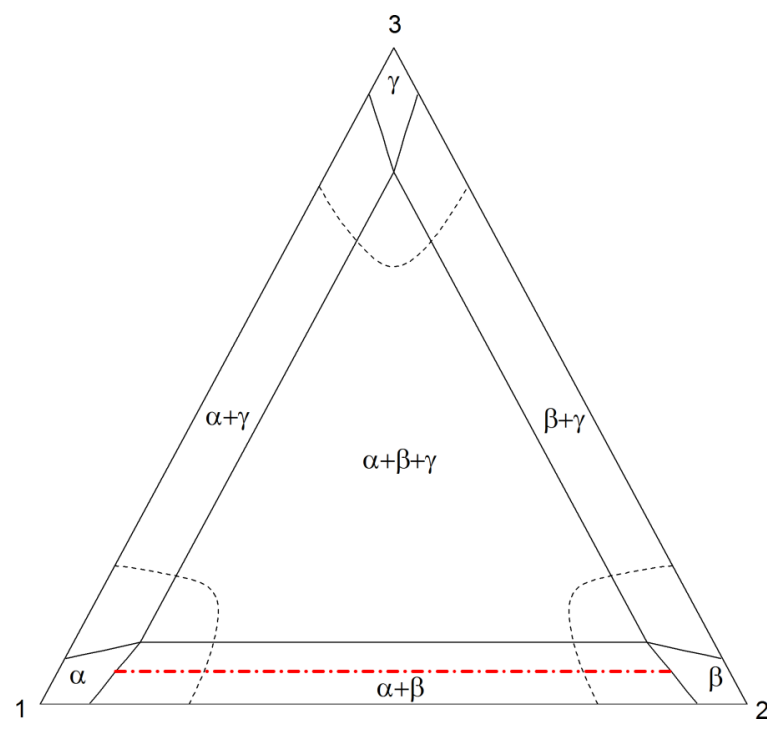

FIG. 2. Phase diagram for the symmetric ternary regular solution with $\eta_{13}$ $=\eta_{23}=\eta_{12}=2 / 3$. System 2 . The dashed line is the intersection of the spinodal surface. For the tie-line shown (red line), the equilibrium compositions are $\bar{c}_{1}^{\alpha}=0.868$ and $\bar{c}_{2}^{\alpha}=0.082$ in $\alpha$ and $\bar{c}_{1}^{\beta}=0.082$ and $\bar{c}_{2}^{\beta}=0.868$ in $\beta$. The spinodal limit is located at $c_{2}^{s}=0.208\left(c_{1}^{s}=0.742\right)$. the string satisfies a differential equation which by construction guarantees that it evolves to the most probable transition pathway connecting two regions $\mathrm{A}$ and $\mathrm{B}$ on both side of the saddle point. The string method requires the initial and final states. The initial state (A) corresponds to the initial supersaturated solution (metastable) and the final state (B) is arbitrary chosen far from the saddle point and is susceptible to move. The initial string is constructed by linear interpolation between the initial and final states. The constraint on compositions (Eq. (3)) is not imposed in the simulations since the small amount of solute atoms incorporated in the growing nucleus is negligible. Note that time is fictitious in the simulation. Images along the string are equally spaced apart in arc length.

\section{MINIMUM FREE ENERGY PATH FOR NUCLEATION}

\section{A. System 1: An asymmetric regular solution}

MFEPs for nucleation were computed for various supersaturations along the same equilibrium tie-line (shown in Fig. 1) from the binodal line up to the spinodal limit. The maximum of the reduced free energy, $\bar{W}$, along the MFEP is the nucleation barrier, i.e., the work required to form the critical nucleus. MFEPs are represented in Fig. 3 for various initial compositions $\left(c_{2}^{0}\right)$. The work to form the critical nucleus is given in Fig. 4 as a function of the initial composition $\left(c_{2}^{0}\right)$. Results are compared to the predictions of the CNT with the interfacial energy (by unit area) defined by Eq. (26). The nucleation barrier was computed from its classical expression, which gives in $4 \pi N_{v} k T \lambda^{3}$ units: $\bar{W}_{c}=\bar{R}^{2} \bar{\sigma} / 3$, with $\bar{R}$ $=R / \lambda=2 \bar{\sigma} / \Delta f_{0}^{\prime}$. The interfacial energy was calculated from the composition profiles for a flat interface (Fig. 8). Classically, the nucleation barrier was found to decrease with the supersaturation. It was found to tend toward infinity near the binodal line, as the driving force for nucleation vanishes and, as found by $\mathrm{CH}$ for the binary case, it vanishes near the spinodal line contrary to the predictions of the classical theory, as shown in Fig. 4, which shows a smooth transition between the binodal and the spinodal regimes. As anticipated, height of the nucleation barrier only agrees with the predictions of the CNT for very low supersaturation.

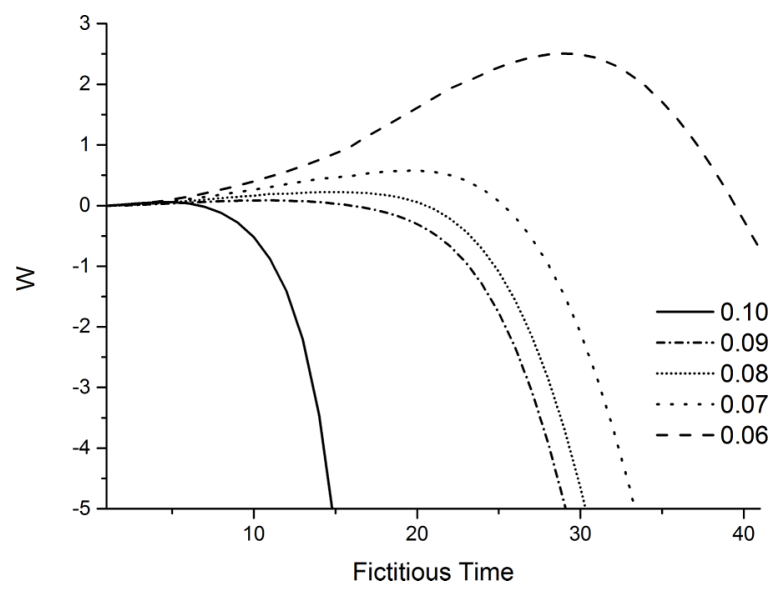

FIG. 3. Nucleation barrier along the minimum free energy path for various initial compositions $\left(c_{2}^{0}\right)$ along the tie-line shown in Fig. 1. System 1. 


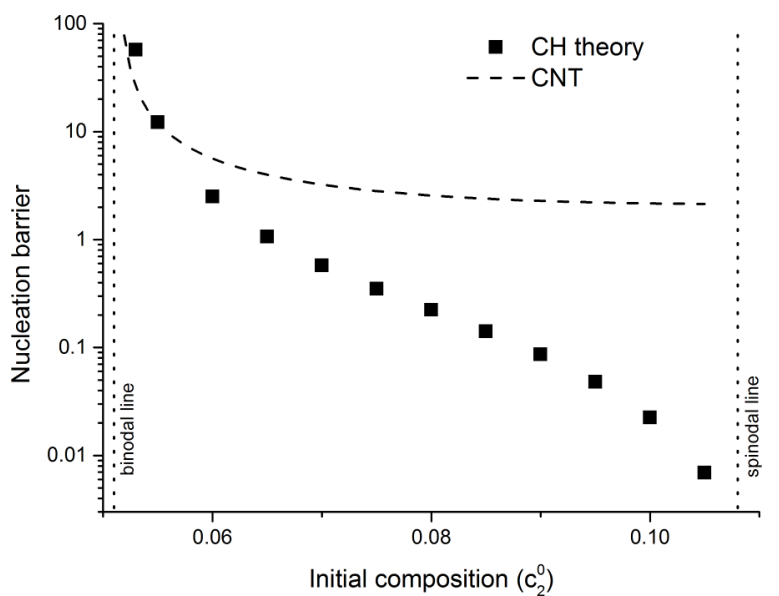

FIG. 4. Work required to form a critical nucleus (expressed in $4 \pi N_{v} k T \lambda^{3}$ units) as a function of the initial composition $c_{2}^{0}$. Results are compared to the predictions of the CNT. System 1. The binodal is located at $\bar{c}_{2}^{\alpha}=0.051$ and the spinodal at $c_{2}^{s}=0.108$.

Radial composition profiles for the critical nucleus, as computed with the string method, are shown in Fig. 5 as a function of the initial composition $\left(c_{2}^{0}\right)$. The molar fraction of specie 1 , at the centre of the nucleus, tends to a composition close to equilibrium for low super-saturation, precisely the composition maximizing the driving force for nucleation. Moreover, as found by $\mathrm{CH}$, the molar fraction of specie 1, at the centre of the nucleus, tends to that of the exterior phase near the spinodal (Fig. 5(a)). Specie 2 was found to behave differently, as shown in Fig. 5(b). Near the spinodal line, the composition at the centre of the critical nucleus still tends to that of the exterior phase; however, for low super-saturation, specie 2 was found to segregate at the interface. Such effect, interpreted as interfacial adsorption, ${ }^{48-50}$ is certainly not pictured by classical nucleation and results (as shown in Sec. IV C) from the particular topology of the driving force. In other words, every other monotonic variations of composition will lead to a higher barrier for nucleation.

In addition, MFEPs also describe the properties of the sub- and super-critical clusters during nucleation. A MFEP, as computed with the string method for a given initial composition, is given in Fig. 6, which shows the successive radial composition profiles during nucleation. Composition profiles of specie 1 (and 3) show a similar two-steps nucleation process to the one found in the binary case: ${ }^{41,42}$ the nucleus rapidly enriches in specie 1 with no significant change in size and then grows with the composition maximizing the driving force for nucleation, which is close to equilibrium. Once again, specie 2 behaves differently (Fig. 6(b)) and exhibits an atypical nucleation scenario: the nucleus first enriches with no change in size until reaching a composition close to equilibrium but once growth starts the composition at its centre slowly decreases and concomitantly specie 2 segregates at the interface.

The composition at the centre of the critical nucleus is shown in Fig. 7 as a function of the initial composition, from the binodal line up to the spinodal limit. The results are compared to the analytical predictions of the classical nucleation theory, recently derived by Philippe et al. ${ }^{51}$ who have determined the composition of the critical cluster $\left(C_{j}^{\beta^{*}}\right)$
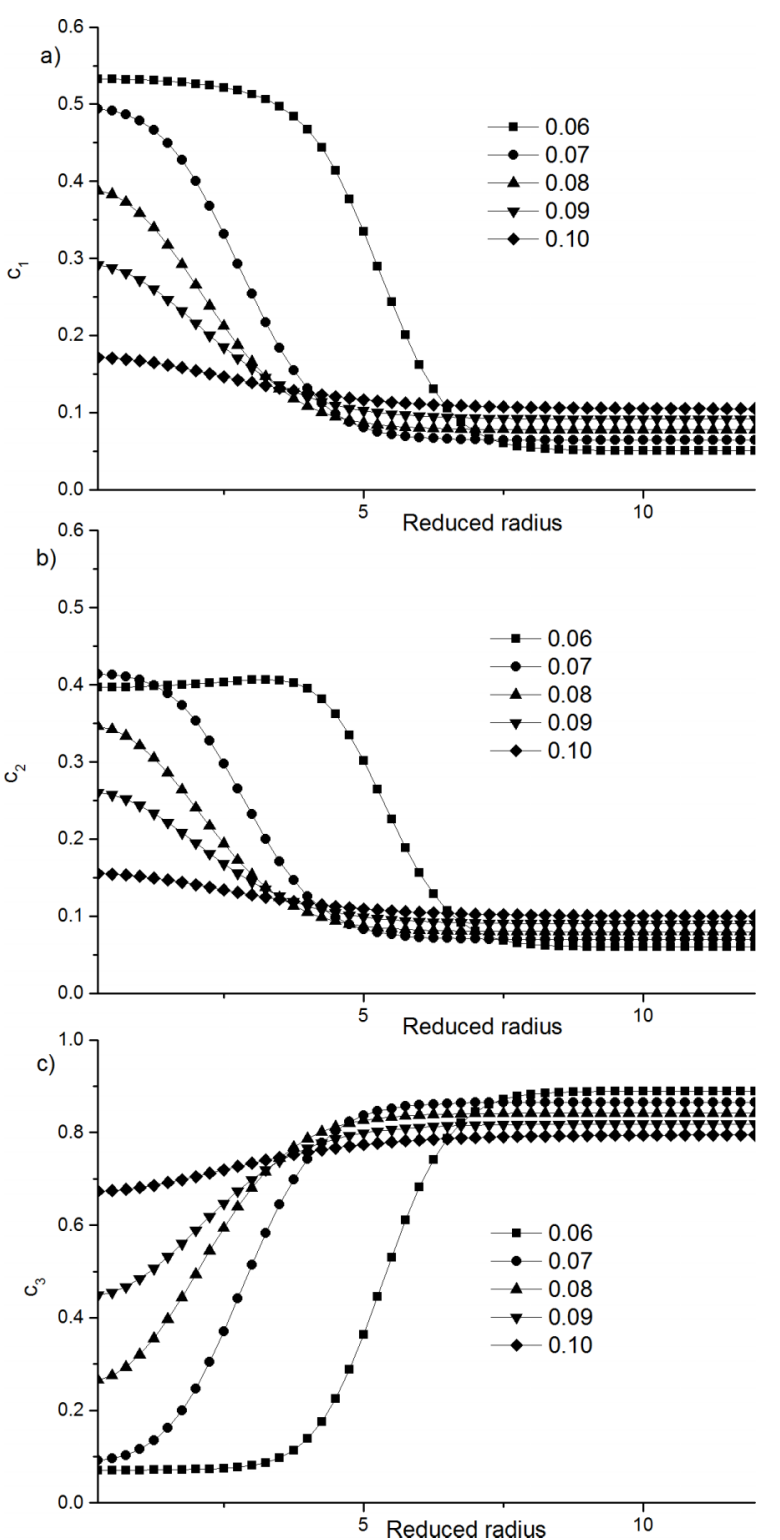

FIG. 5. Radial composition profiles of the critical nucleus for different initial compositions $c_{2}^{0}$.

in the limit of small supersaturation,

$$
\Delta \mathbf{C}^{\beta^{*}}=\mathrm{F}^{\beta-1} \mathrm{~F}^{\alpha} \Delta \mathbf{C}^{0}
$$

for same partial molar volume. $\Delta \mathbf{C}^{\beta^{*}}=C_{j}^{\beta^{*}}-\bar{C}_{j}^{\beta}$ and $\Delta \mathbf{C}^{0}$ $=C_{j}^{0}-\bar{C}_{j}^{\alpha}$ for $\mathrm{j}=1,2$ and $\mathrm{F}^{\alpha}$ and $\mathrm{F}^{\beta}$ are the Hessians of the free energy evaluated at $\bar{C}_{j}^{\alpha}$ and $\bar{C}_{j}^{\beta}$, respectively. Nucleus compositions only agree with predictions of classical nucleation for low super-saturation. This reveals that classical nucleation theory fails to properly describe nucleation for high supersaturation. Still for low super-saturation, molar fraction of specie 1 is higher than its equilibrium value, due to capillarity (interface curvature), and this deviation in composition from equilibrium increases with super-saturation, as predicted by classical nucleation. Molar fraction of specie 2 at the centre of the critical nucleus is, contrary to specie 1 , lower than its equilibrium value and decreases with the super-saturation, as also predicted by CNT. Such deviations from equilibrium are 


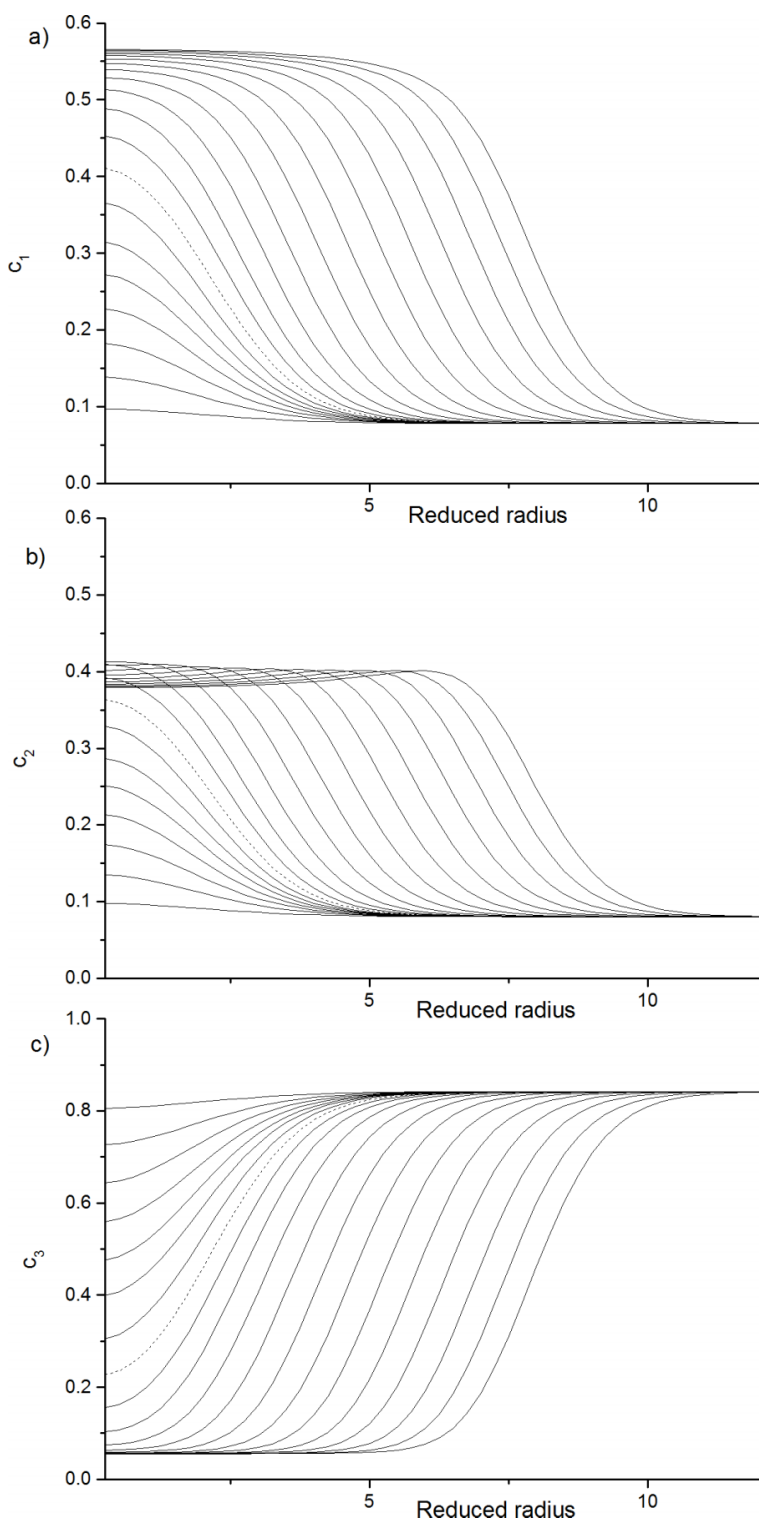

FIG. 6. Radial composition profiles along the MFEP for a given initial composition $c_{2}^{0}=0.08$. The critical nucleus (saddle point) is plotted in dashed lines.

related to the curvature dependence of the free energy ${ }^{51}$ and make the composition of the critical nucleus to not lie on the equilibrium tie-line when the Hessians of the free energies evaluated at equilibrium compositions are not identical. Finally, both compositions were found to tend to that of the exterior phase near the spinodal region.

Then, the Euler equation (Eq. (19)) is solved numerically and composition profiles of a flat interface are determined, which are then used to compute the free energy of a flat interface (Eq. (26)). The composition profiles leading to an extrema in the free energy are the profiles of the actual interface and are shown in Fig. 8. The initial composition lies on the tie-line shown in Fig. 1. Thus, the equilibrium compositions correspond to those defining this tie-line. The interfacial composition profiles exhibit, as in nucleation, a core-shell structure, with a higher molar fraction for specie 2 in the interfacial region, as compared with equilibrium. The way the composition $c_{2}$ changes with $c_{1}$ through the interface

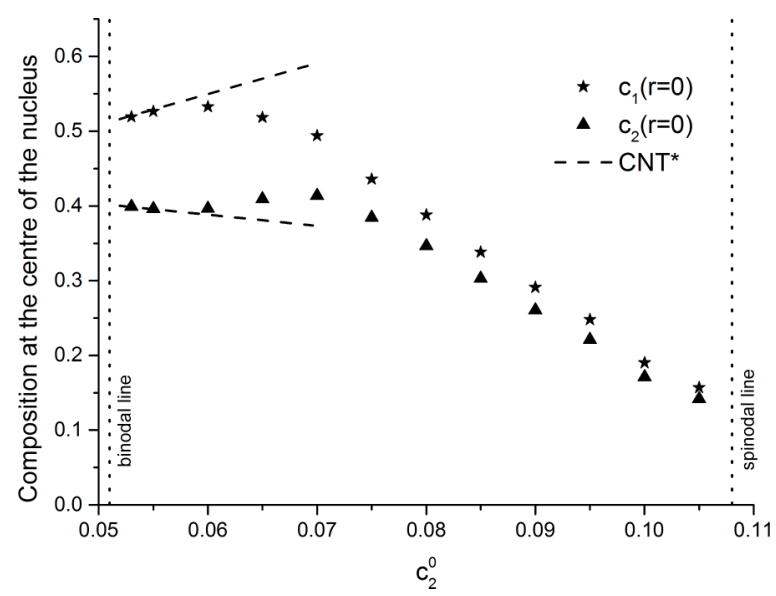

FIG. 7. Composition at the centre of the critical nucleus for various initial compositions $c_{2}^{0}$. The results are compared to the analytical predictions of the classical nucleation theory valid for low supersaturation only $\left(\mathrm{CNT}^{* 51}\right)$. The binodal is located at $\bar{c}_{2}^{\alpha}=0.051$ and the spinodal at $c_{2}^{s}=0.108$. The equilibrium compositions in the $\beta$ phase are $\bar{c}_{1}^{\beta}=0.514$ and $\bar{c}_{2}^{\beta}=0.401$.

was found to be non-monotonic. This phenomenon, known as interfacial adsorption, ${ }^{48-50}$ is well understood for flat interfaces at equilibrium and was found to lower the interfacial energy and increases the interfacial thickness. ${ }^{48-50}$ As suggested by Cahn and Hilliard, the way $c_{2}$ changes with $c_{1}$ along the flat interface in ternary systems does not strictly follow the free energy $\left(\Delta \bar{f}_{0}^{\prime}\right)$ so that to reduce the gradient energy at the expense of the volume energy. However, it will be shown in Sec. IV C that the actual trajectory remains sensitive to the topology of $\Delta \bar{f}_{0}^{\prime}$ and thus to complex changes in curvatures.

\section{B. System 2: The symmetric regular solution}

In this section, nucleation in symmetric regular solution is investigated. The reduced temperatures are set to $2 / 3$. Initial compositions lie on the tie-line shown in Fig. 2. The third component is minority with the same equilibrium composition in both phases $\left(\bar{c}_{3}=0.05\right)$, i.e., no partition. Of interest is to determine how the minority component is sensitive to nucleation despite the fact it is non-selective (no partition). The string method is used to compute MFEPs for nucleation. The radial composition profiles of the critical nucleus (saddle point)

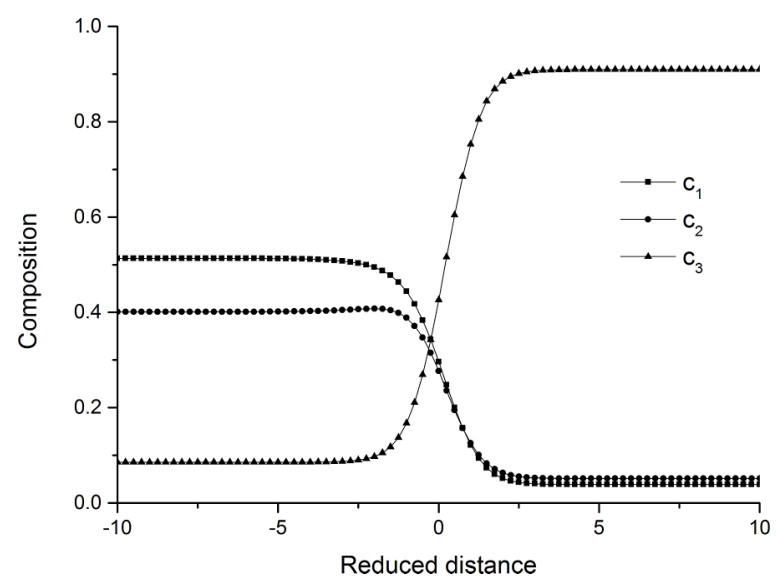

FIG. 8. Composition profiles across a flat interface. System 1. 


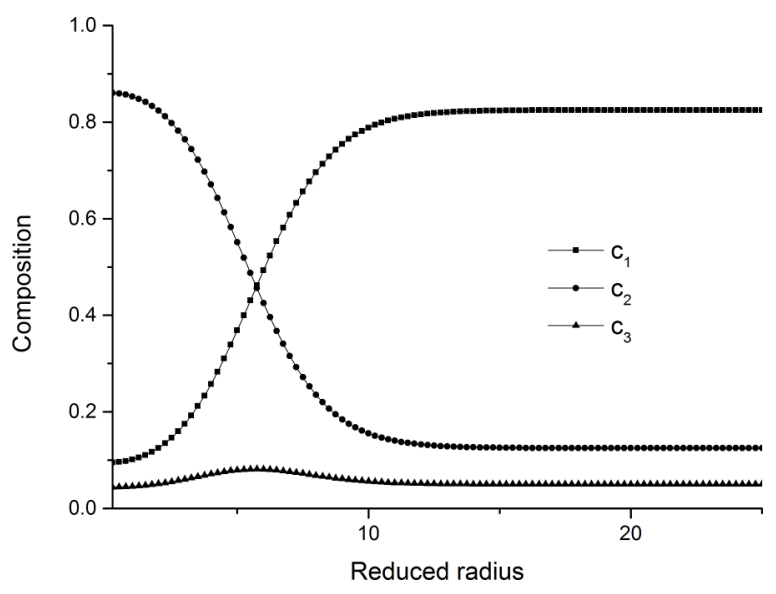

FIG. 9. Radial composition profiles of the critical nucleus for a given initial composition $c_{2}^{0}=0.125$. System 2 .

are shown in Fig. 9 for a given initial composition $\left(c_{1}^{0}=0.825\right.$ and $c_{2}^{0}=0.125$ ). The results of Fig. 9 illustrate that the minority component segregates at the interface. Interfacial adsorption is clearly visible in Fig. 10, which shows the composition profile of the critical nucleus for the minority component, and for different supersaturations along the tie-line of Fig. 2. For high supersaturation, the minority component shows no segregation at the interface. However the results of Fig. 10 exhibit, as the supersaturtion decreases, adsorption of the minority component at the interface. The critical nucleus also shows, for low supersaturation, a core depleted in minority component (see Fig. 10), as compared with the initial composition $\left(c_{3}^{0}=0.05\right)$.

The successive radial composition profiles along the MFEP, for a given supersaturation, are shown in Fig. 11. As the nucleus grows, the minority component segregates at the interface and the core of the nucleus depletes. Adsorption is visible when the core of the nucleus is clearly developed. This is the case for the critical nucleus near the binodal (Fig. 9). However, the critical cluster near the spinodal is very diffuse and shows no adsorption (Fig. 10). Adsorption at the interface as well as depletion of the core shall decrease the free energy. In order to illustrate this, the composition profiles for a flat interface in equilibrium have been computed by solving the Euler equation and are shown in Fig. 12. Equilibrium

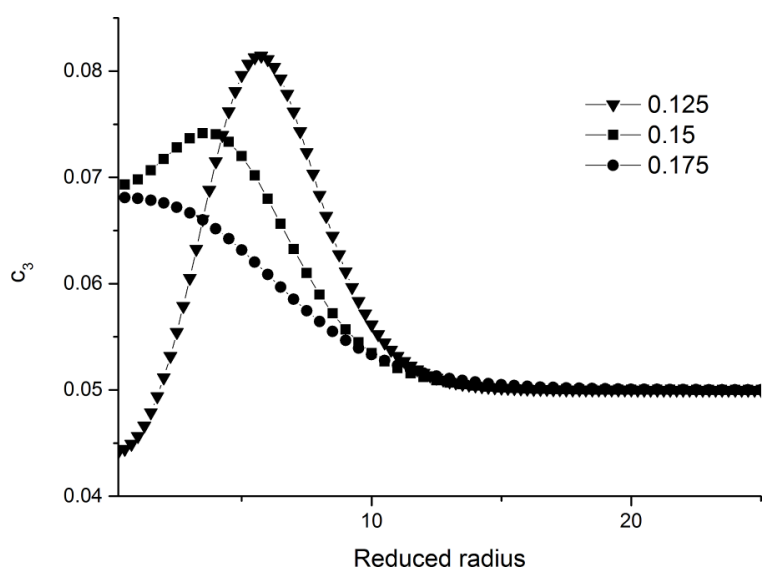

FIG. 10. Radial composition profiles of the critical nucleus for different initial compositions $c_{2}^{0}$ along the tie-line shown in Fig. 2. System 2.

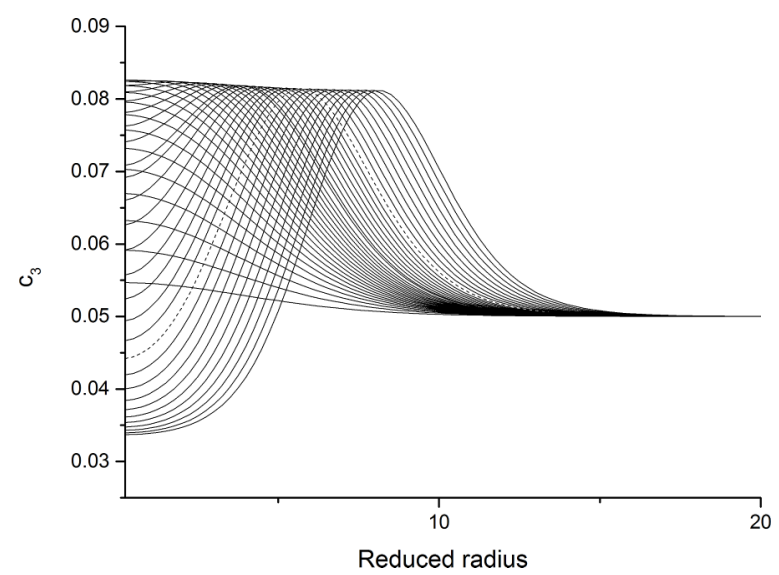

FIG. 11. Radial composition profiles (specie 3) along the MFEP for a given initial composition $c_{2}^{0}=0.125$. The critical nucleus is plotted in dashed line.

compositions are $\bar{c}_{1}^{\alpha}=0.824, \bar{c}_{2}^{\alpha}=0.0916, \bar{c}_{3}^{\alpha}=0.0844$ in $\alpha$ and $\bar{c}_{1}^{\beta}=0.0916, \bar{c}_{2}^{\beta}=0.824, \bar{c}_{3}^{\beta}=0.0844$ in $\beta$. As found in Ref. 50 , the minority component segregates at the interface. The way $c_{2}$ changes with $c_{1}$ is analyzed in Fig. 13. Three different trajectories are represented. One of them is the trajectory of steepest descent along the energy difference between the free energy surface and the equilibrium plane $\left(\Delta \bar{f}_{0}^{\prime}\right.$, Eq. (16)); this shall be the actual trajectory if no gradient energy (abrupt interface). The second trajectory is the one of the actual interface, solution of the Euler equation, see Fig. 12. The case with no adsorption of the minority component constitutes the third trajectory. If it is evident that the way $c_{2}$ changes with $c_{1}$ does not strictly follow the free energy $\Delta \bar{f}_{0}^{\prime}$ but banks on curve to reduce gradient energy at the expense of volume energy. However, the results of Fig. 13 clearly illustrate that the actual interface is strongly influenced by the topology of $\Delta \bar{f}_{0}^{\prime}$.

\section{Interfacial adsorption}

In nucleation, the way compositions evolve along the interface is related to the driving force by the Euler-Lagrange equation (Eq. (9)). Figure 14 shows the driving force $\Delta f_{0}^{\prime}$ as

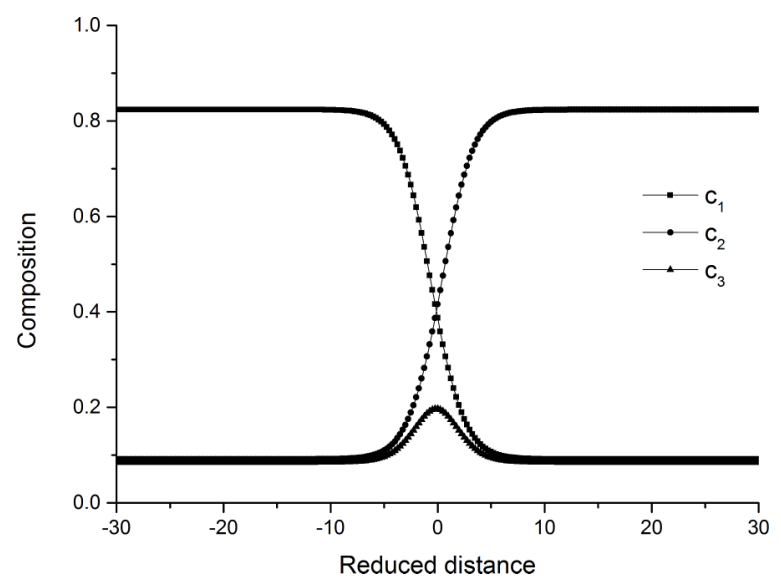

FIG. 12. Composition profiles across a flat interface for the symmetric regular solution. Equilibrium compositions are $\bar{c}_{1}^{\alpha}=0.824, \bar{c}_{2}^{\alpha}=0.0916, \bar{c}_{3}^{\alpha}$ $=0.0844$ in $\alpha$ and $\bar{c}_{1}^{\beta}=0.0916, \bar{c}_{2}^{\beta}=0.824, \bar{c}_{3}^{\beta}=0.0844$ in $\beta$. 


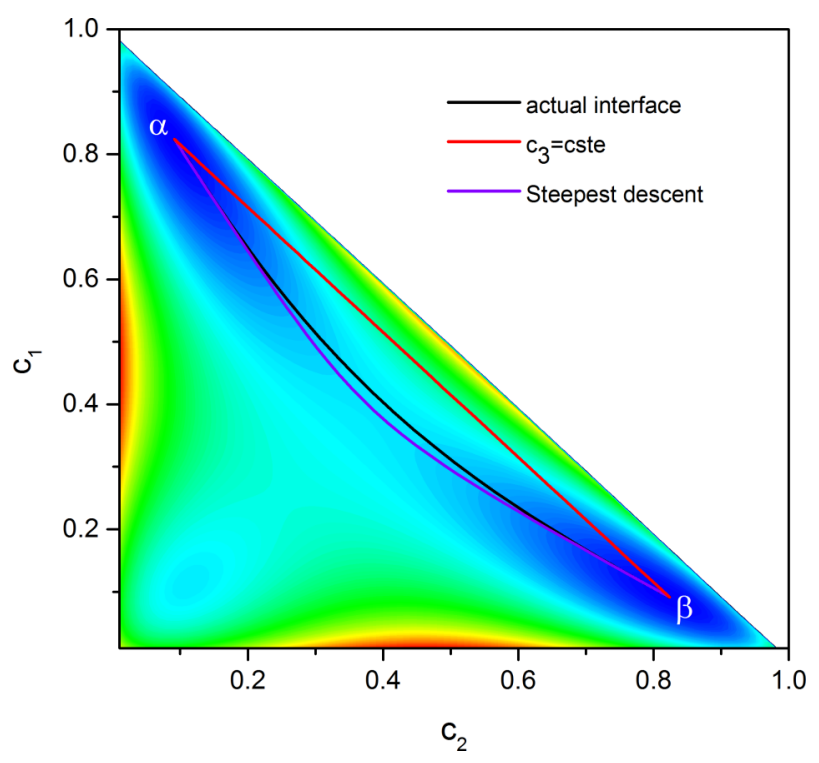

FIG. 13. Energy difference between the free energy of the symmetric ternary regular solution and the equilibrium plane for a given nominal composition. The color scale indicates the magnitude of $\Delta \bar{f}_{0}^{\prime}$, from red (maximum of the function) to blue (minimum). The ways $c_{2}$ changes with $c_{1}$, as given by the steepest descent (saddle point, no gradient energy), by the actual interface (solution of the Euler equation, see Fig. 10) and for no adsorption of the minority component, are also represented. System 2.

a function of compositions for the symmetric regular solution $(\eta=2 / 3)$ and for low supersaturation $\left(c_{2}^{0}=0.098\right)$. The way $c_{2}$ changes with $c_{1}$ along the actual interface (saddle point of the free energy, Eq. (25)) is compared with the case with no adsorption of the minority component. The potential surface indicates that segregation of the minority component increases (in absolute value) the driving force for nucleation. As for the planar interface, the way $c_{2}$ changes with $c_{1}$ banks on the

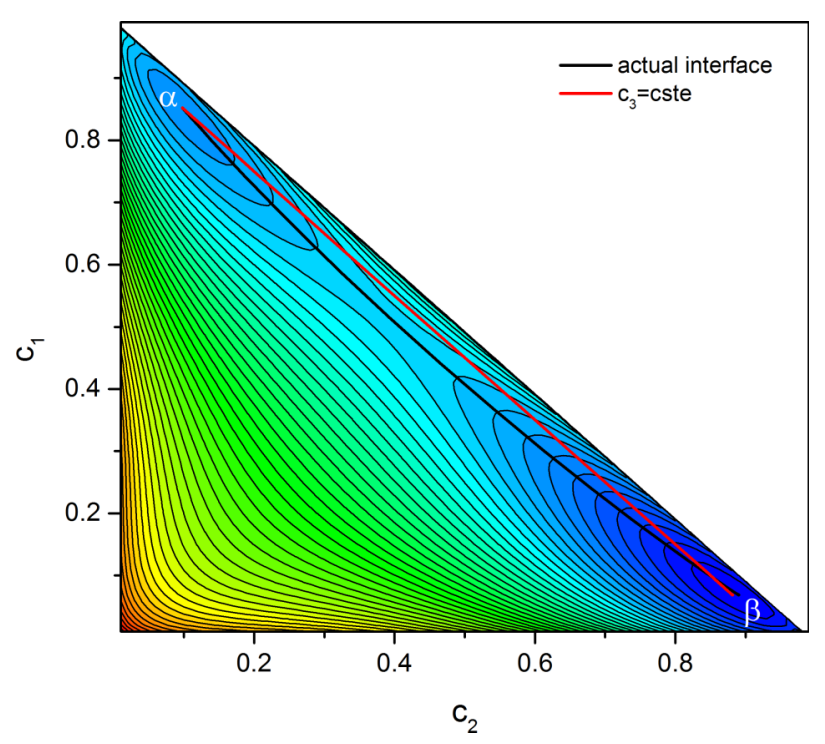

FIG. 14. Contour lines of the driving force for nucleation for a given nominal composition $\left(c_{2}^{0}=0.098\right)$. The color scale indicates the magnitude of $\Delta f_{0}^{\prime}$, from red (maximum of the function) to blue (minimum). The ways $c_{2}$ changes with $c_{1}$ as given by the actual interface are compared to the no segregation case. System 2. driving force to reduce the gradient energy but remains very close to the steepest descent.

A Landau-type analysis in the limit of small adsorption (and for $c_{1}^{0}=c_{2}^{0}$ ) has shown that the minority and non-selective component segregates at the interface so that to decrease the interfacial energy of a flat interface. ${ }^{49,50} \mathrm{~A}$ similar Landau-type analysis appears difficult in the present case for several reasons, such as large degrees of adsorption. Moreover, the interface is under pressure in the present case. Thus, the compositional gradient terms have a strong influence on the interfacial properties and the mentioned analysis does not apply. However, such segregation of the minority component can be qualitatively understood from the Euler-Lagrange condition, which relates the composition profiles and the driving force for nucleation. In Secs. IV A and IV B, the free energy of the regular solution was a function of the two independent compositions $c_{1}$ and $c_{2}$. This choice was arbitrary, and if the minority component $c_{3}$ is chosen as one of the independent compositions, the composition profile in minority component must also satisfy the Euler-Lagrange

$$
\frac{2}{\eta} \frac{d^{2} c_{3}}{d r^{2}}+\frac{4}{\eta r} \frac{d c_{3}}{d r}=\frac{\partial \Delta f_{0}^{\prime}\left(c_{1}, c_{3}\right)}{\partial c_{3}},
$$

where $\Delta f_{0}^{\prime}$ is now a function of $c_{1}$ and $c_{3}$. Thus, no segregation of the minority component implies, systematically, that the derivative of the driving force with respect to the minority component along the nucleus interface equals zero, i.e., $\partial \Delta f_{0}^{\prime}\left(c_{1}, \bar{c}_{3}\right) / \partial c_{3}=0$. If the interfacial composition profile with no adsorption of the minority component, i.e., $c_{3}(\bar{r})$ $=c_{3}^{0}=\bar{c}_{3}$, does not satisfy the Euler condition, it is obviously not an extrema of the nucleation barrier. Fig. 15 shows the derivative of the driving force with respect to the minority component along the nucleus interface, for the case with no segregation $\left(c_{3}=\bar{c}_{3}\right)$. As this derivative does not equal zero, the composition profiles with no adsorption are not an extrema of the free energy as it does not satisfy the Euler-Lagrange equation. Consequently, the molar fraction of the minority component must be non-uniform. This does not mean that this non-uniformity systematically involves $\partial \Delta f_{0}^{\prime} / \partial c_{3}=0$ along the actual profile.

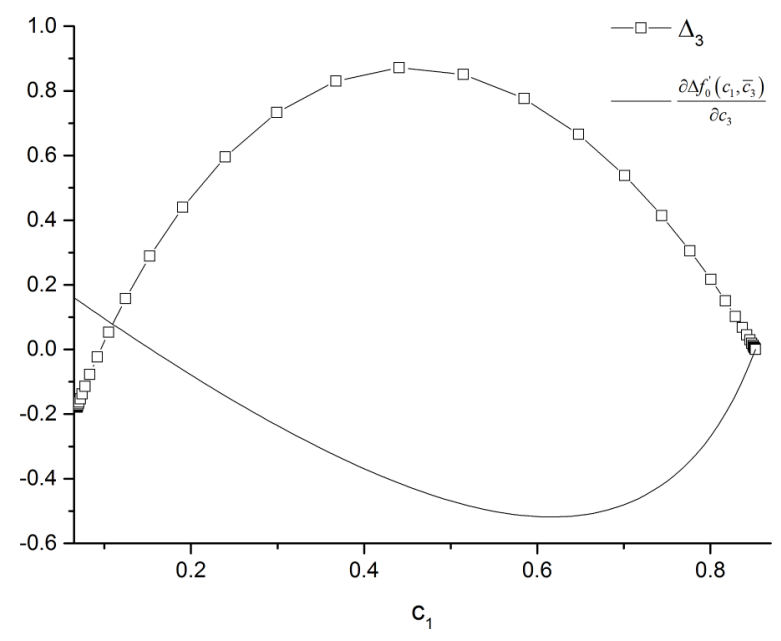

FIG. 15. The function $\partial \Delta f_{0}^{\prime}\left(c_{1}, \bar{c}_{3}\right) / \partial c_{3}$ and the segregation of the minority component for the critical nucleus (shown in Fig. 16): $\Delta_{3}=\left(c_{3}-\bar{c}_{3}\right) / \bar{c}_{3}$. 


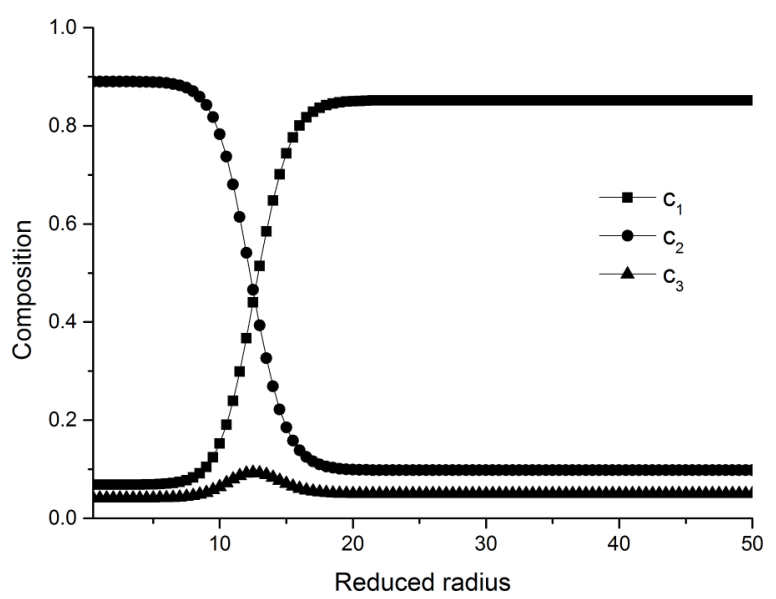

FIG. 16. Radial composition profiles of the critical nucleus for $c_{2}^{0}=0.098$.

The way $c_{2}$ (or $c_{3}$ ) changes with $c_{1}$ is qualitatively pictured by $\partial \Delta f_{0}^{\prime}\left(c_{1}, \bar{c}_{3}\right) / \partial c_{3}$. Figure 15 compares the segregation of the minority component (see Fig. 16) with this function. Segregation of the minority component coincides with $\partial \Delta f_{0}^{\prime}\left(c_{1}, \bar{c}_{3}\right) / \partial c_{3}<0$ and depletion with $\partial \Delta f_{0}^{\prime}\left(c_{1}, \bar{c}_{3}\right) / \partial c_{3}$ $>0$. The composition profile $c_{3}$ of the critical nucleus (shown in Fig. 16) shows inhomogeneity so that to decrease the free energy $\Delta f_{0}^{\prime}\left(c_{1}, c_{3}\right)$. The results of Fig. 16 qualitatively illustrate that the minority component segregates at the interface when increasing $c_{3}$ decreases the free energy, and the core of the nucleus depletes when decreasing the composition decreases the free energy.

\section{CONCLUSION}

Nucleation has been studied in ternary systems by examining the topology of the overall landscape of the energy surface in the framework of the continuum theory. The MFEP for nucleation of a single nucleus in an infinite matrix was computed for an asymmetric and for the symmetric ternary regular solution. MFEPs were computed with the string method and were found to exhibit complex nucleation pathways. It is shown that the complex interplay between the volume energy, i.e., driving force for nucleation, and the gradient energy can lead to very different nuclei, such as core-shell structures. Such interfacial segregation phenomena and the unpredicted partitions of non-selective components are expected to affect nucleation kinetics.

\section{ACKNOWLEDGMENTS}

I am grateful for the financial support of CARNOT ESP Institute and I wish to thank Professor P. W. Voorhees for important discussions on interfacial adsorption.

\section{APPENDIX A: THE FREE ENERGY OF A NON-UNIFORM SYSTEM}

Following $\mathrm{CH},{ }^{13}$ we expand the local free energy, $f$, about the free energy of the homogeneous solution, $f_{0}^{N}$,

$$
\begin{aligned}
f= & f_{0}^{N}\left(c_{1}, c_{2}, \ldots, c_{N}\right)+\left.\sum_{i=1}^{N} \nabla c_{i} \frac{\partial f_{0}^{N}}{\partial \nabla c_{i}}\right|_{c_{1}, c_{2}, \ldots, c_{N}} \\
& +\left.\sum_{i=1}^{N} \nabla^{2} c_{i} \frac{\partial f_{0}^{N}}{\partial \nabla^{2} c_{i}}\right|_{c_{1}, c_{2}, \ldots, c_{N}} \\
& +\left.\frac{1}{2 !} \sum_{i=1}^{N} \sum_{j=1}^{N} \nabla c_{i} \nabla c_{j} \frac{\partial^{2} f_{0}^{N}}{\partial \nabla c_{i} \partial \nabla c_{j}}\right|_{c_{1}, c_{2}, \ldots, c_{N}}+\cdots
\end{aligned}
$$

Similar Taylor expansions have been proposed in multicomponent systems by several authors. ${ }^{45-47}$ For isotropic systems or crystal structures with cubic symmetry, the second term of the expansion vanishes, ${ }^{13}$ thus

$$
f=f_{0}^{N}\left(c_{1}, c_{2}, \ldots, c_{N}\right)+\sum_{i=1}^{N} \bar{\Gamma}_{i} \nabla^{2} c_{i}+\sum_{i=1}^{N} \sum_{j=1}^{N} \bar{\xi}_{i j} \nabla c_{i} \nabla c_{j},
$$

with

$$
\bar{\Gamma}_{i}=\left.\frac{\partial f_{0}^{N}}{\partial \nabla^{2} c_{i}}\right|_{c_{1}, c_{2}, \ldots, c_{N}}
$$

and

$$
\bar{\xi}_{i j}=\left.\frac{1}{2} \frac{\partial^{2} f_{0}^{N}}{\partial \nabla c_{i} \partial \nabla c_{j}}\right|_{c_{1}, c_{2}, \ldots, c_{N}} .
$$

Using the sum rule (Eq. (1)) in the local free energy leads to

$$
\begin{aligned}
f= & f_{0}\left(c_{1}, c_{2}, \ldots, c_{N-1}\right) \\
& +\sum_{i=1}^{N-1} \Gamma_{i} \nabla^{2} c_{i}+\sum_{i=1}^{N-1} \sum_{j=1}^{N-1} \xi_{i j} \nabla c_{i} \nabla c_{j},
\end{aligned}
$$

where

$$
\Gamma_{i}=\bar{\Gamma}_{i}-\bar{\Gamma}_{N}
$$

and

$$
\xi_{i j}=\bar{\xi}_{i j}-\bar{\xi}_{N j}-\bar{\xi}_{i N}+\bar{\xi}_{N N}
$$

The bulk Helmholtz free energy, $F$, of the solution is given by the integral of the local free energy over the volume of the system. By using the divergence theorem, $F$ is simplified to

$F=\int_{V}\left\{f_{0}\left(c_{1}, c_{2}, \ldots, c_{N-1}\right)+\sum_{i=1}^{N-1} \sum_{j=1}^{N-1} \kappa_{i j} \nabla c_{i} \nabla c_{j}\right\} d V$,

where the $\kappa_{i j}$ are the gradient energy coefficients

$$
\kappa_{i j}=\xi_{i j}-\delta_{i j} \frac{\partial \Gamma_{j}}{\partial c_{i}},
$$

and are symmetrical and composition independent. $\delta_{i j}$ is the Kronecker symbol. Equation (A8) is the extension of the $\mathrm{CH}$ free energy of an inhomogeneous solution for a multicomponent system. ${ }^{45}$

\section{APPENDIX B: THE GRADIENT ENERGY COEFFICIENTS OF THE REGULAR SOLUTION}

We extend the procedure of $\mathrm{CH}^{13}$ and $\mathrm{Hoyt}^{45}$ to express the gradient energy coefficients in a multicomponent regular 
solution. It is required to compute all the $P_{i j}$ (for $j>i$ ) probabilities of forming an $i j$ bond by an $i$ atom at site $R$ and a $j$ atom at site $S$. As an illustration, the probability $P_{1 N}$ is given by

$$
P_{1 N}=c_{1}(R) c_{N}(S)=c_{1}(R)\left(1-\sum_{i=1}^{N-1} c_{i}(S)\right) .
$$

We expand $c_{i}(S)$ about $R$ to second order

$$
c_{i}(S)=c_{i}(R)+(r . \nabla) c_{i}(R)+(1 / 2 !)(r . \nabla)^{2} c_{i}(R),
$$

where $r$ is the radius vector of site $S$ relative to $R$. The probable number of $1 N$ bonds, $n_{1 N}$, between an atom of specie 1 at site $R$ and an atom of specie $\mathrm{N}$ in its nth shell (at $r_{n}$ ) is for a cubic lattice, ${ }^{13}$

$$
n_{1 N}=Z_{n} c_{1}(R)\left(1-\left(r_{n}^{2} / 6\right) \sum_{i=1}^{N-1} \nabla^{2} c_{i}(R)\right) .
$$

Multiplying the $n_{i j}$ by the order energy $\varepsilon_{i j}^{n}$ between species $i$ and $j$ in the nth coordination shell, summing over $i$ and $j$ gives the energy of mixing per atom relative to the pure component. Comparing the result to Eq. (A1), see Ref. 45 for more details, the expression of the gradient energy coefficients in a multicomponent regular solution can be written as

$$
\kappa_{i j}=\delta_{i j} N_{v} k T_{c}^{i j} \lambda_{i j}^{2},
$$

where $\lambda_{i j}$ are effective interaction distances $\left(\lambda_{i j}=\sum_{n} Z_{n} v_{i j}^{n} r_{n}^{2} /\right.$ $\left.6 k T_{c}^{i j}\right), N_{v}$ is the number of atoms by unit volume, and $\delta_{i j}$ is the Kronecker symbol.

\section{APPENDIX C: THE STRING METHOD}

The string method is a numerical approach for computing transition pathways and free energy barriers. ${ }^{29,30}$ The method proceeds by evolving strings whose dynamics take them to the most probable transition pathway between two metastable regions in configuration space. The metastable states are localized around the minima of the surface energy $W$. Strings are smooth curves with intrinsic parameterization and the string satisfies a differential equation which by construction guarantees that it evolves to the most probable transition pathway. Consider a system modeled by

$$
\gamma \frac{\partial q}{\partial t}=-\nabla W(q)+\xi(t)
$$

which guarantees by construction that the string is the MFEP connecting the metastable regions on both side of the saddle point. In Eq. (1), $\gamma$ is a friction coefficient and $q$ denotes the generalized coordinates. In the context of nucleation in $\mathrm{N}$ components system, the generalized reaction coordinates correspond to the $\mathrm{N}-1$ radial composition profiles. $\xi(t)$ is the white noise satisfying $\langle\xi i(t) \xi j(t)\rangle=2 k T \gamma \delta i j \delta\left(t-t^{\prime}\right)$. The MFEP is the smooth curve $\varphi^{*}$ satisfying

$$
(\nabla W)^{\perp}\left(\varphi^{*}\right)=0,
$$

where $(\nabla W)^{\perp}$ is the component of $\nabla W$ normal to $\varphi^{*}$. An initial string $\varphi$ is considered, not necessarily the $\operatorname{MFEP} \varphi^{*}$, that connects the two metastable regions. $\varphi(\alpha, t)$ represents the instantaneous position of the string at a given time with $\alpha$ a suitable parameterization. Thus, a simple method to find the MFEP is to make $\varphi$ evolve according to the normal velocity of $\varphi$,

$$
\frac{\partial \varphi}{\partial t}=-[\nabla W(\varphi)]^{\perp}+s u
$$

where time is renormalized $(t / \gamma \rightarrow t),(\nabla W)^{\perp}=\nabla W-(\nabla W . u)$ $u$, and $u$ is the unit tangent vector along $\varphi . s$ is a Lagrange multiplier determined by the choice of parameterization. $\varphi$ was here parameterized through the normalisation of the arc length. The string method proceeds in two steps: the string is discretized into a finite number of points which move according to the normal component of the force $-[\nabla W(\varphi)]^{\perp}$. Then, a reparametrization step is done to reinforce $\left(\left|\varphi_{\alpha}\right|\right)_{\alpha}=0 .{ }^{29}$

${ }^{1}$ J. W. Gibbs, Trans. Connecticut Acad. Sci. 3(108), 343 (1875-1878); J. W. Gibbs, The Collected Works (Longmans Green, New York, 1928), Vol. 1.

${ }^{2}$ J. D. van der Waals and $\mathrm{Ph}$. Kohnstamm, Lehrbuch der Thermodynamik (Johann- Ambrosius-Barth, Leipzig, 1908).

${ }^{3}$ J. S. Rowlinson, J. Stat. Phys. 20, 197 (1979) [translated by J. D. Van der Waals, Z. Phys. Chem. 13, 657 (1893) (in German)].

${ }^{4}$ M. Volmer, Kinetik der Phasenbildung (Steinkopff, Dresden, 1939).

${ }^{5}$ Y. I. Frenkel, Kinetic Theory of Liquids (Oxford University Press, Oxford, 1946).

${ }^{6}$ Nucleation, edited by A. C. Zettlemoyer (Marcel Dekker, New York, 1969).

${ }^{7}$ A. C. Zettlemoyer, Adv. Colloid Interface Sci. 7, vii (1977).

${ }^{8}$ J. D. Gunton, M. S. Miguel, and P. S. Sahni, in Phase Transitions and Critical Phenomena, edited by C. Domb and J. L. Lebowitz (Academic Press, London, 1983), Vol. 8.

${ }^{9}$ V. P. Skripov, Metastable Liquids (Wiley, New York, 1974) [Nauka, Moscow, 1972 (in Russian)].

${ }^{10}$ R. Becker and W. Doring, Ann. Phys. 416, 719 (1935).

${ }^{11}$ Zeldovich, J. Exp. Theor. Phys. USSR 12, 525 (1942).

${ }^{12}$ D. Turnbull and J. C. Fisher, J. Chem. Phys. 17, 71 (1949).

${ }^{13}$ J. W. Cahn and J. E. Hilliard, J. Chem. Phys. 28, 258 (1959).

${ }^{14}$ J. W. Cahn and J. E. Hilliard, J. Chem. Phys. 31, 688 (1959).

${ }^{15}$ F. Schmitz, P. Virnau, and K. Binder, Phys Rev. E 87, 053302 (2013).

${ }^{16}$ L. Maragliano, A. Fischer, E. Vanden-Eijnden, and G. Ciccotti, J. Chem. Phys. 125, 024106 (2006)

${ }^{17}$ M. Venturoli, E. Vanden-Eijnden, and G. Ciccotti, J. Math. Chem. 45, 188 (2009).

${ }^{18}$ M. I. Freidlin and A. D. Wentzell, Random Perturbations of Dynamical Systems (Springer, New York, Verlag, Berlin, 1984).

${ }^{19}$ V. G. Baidakov, G. S. Boltashev, and J. W. P. Schmelzer, J. Colloid Interface Sci. 231, 312 (2000).

${ }^{20}$ J. W. P. Schmelzer, J. Schmelzer, and I. Gutzow, J. Chem. Phys. 112, 3820 (2000).

${ }^{21}$ T. Philippe and D. Blavette, Philos. Mag. 91, 4606-4622 (2011).

${ }^{22}$ J. W. P. Schmelzer, A. R. Gokhman, and V. M. Fokin, J. Colloid Interface Sci. 272, 109 (2004)

${ }^{23}$ C. L. Weakliem and H. Reiss, J. Chem. Phys. 99, 5374 (1993).

${ }^{24}$ H. Trinkaus, Phys. Rev. B 27, 7372 (1983).

${ }^{25}$ M. Iwamatsu, J. Chem. Phys. 140, 064702 (2014).

${ }^{26}$ T. Philippe and P. W. Voorhees, Acta Mater. 61, 4237 (2013).

${ }^{27}$ G. Henkelman and H. Jónsson, J. Chem. Phys. 113, 9978 (2000).

${ }^{28}$ G. Henkelman, B. P. Uberuaga, and H. Jónsson, J. Chem. Phys. 113, 9901 (2000).

${ }^{29}$ W. E, W. Ren, and E. Vanden-Eijnden, Phys. Rev. B 66, 052301 (2002).

${ }^{30}$ W. E, W. Ren, and E. Vanden-Eijnden, J. Chem. Phys. 126, 164103 (2007).

${ }^{31}$ M. Iwamatsu, J. Chem. Phys. 130, 244507 (2009).

${ }^{32}$ J. F. Lutsko, J. Chem. Phys. 128, 184711 (2008).

${ }^{33}$ J. F. Lutsko, J. Chem. Phys. 129, 244501 (2008).

${ }^{34}$ S. M. Wood and Z.-G. Wang, J. Chem. Phys. 116, 2289 (2002).

${ }^{35}$ C.-Z. Zhang and Z.-G. Wang, Phys. Rev. E 77, 021906 (2008).

${ }^{36}$ C. Qiu, T. Qian, and W. Ren, J. Chem. Phys. 129, 154711 (2008).

${ }^{37}$ X. Cheng, L. Lin, W. E. P. Zhang, and A.-C. Shi, Phys. Rev. Lett. 104, 148301 (2010).

${ }^{38}$ R. Backofen and A. Voigt, J. Phys.: Condens. Matter 22, 364104 (2010).

${ }^{39}$ Q. Du and L. Zhang, Commun. Math. Sci. 7, 1039 (2009). 
${ }^{40}$ L. Zhang, L. Q. Chen, and Q. Du, J. Comp. Phys. 229, 6574 (2010).

${ }^{41}$ T. Philippe and D. Blavette, Scripta Mater. 67, 77-80 (2012).

${ }^{42}$ T. Philippe and D. Blavette, J. Chem. Phys. 135, 134508 (2011).

${ }^{43}$ W. Ren, Langmuir 30, 2879-2885 (2014).

${ }^{44} \mathrm{Y}$. Li and W. Ren, Langmuir 30, 9567-9576 (2014).

${ }^{45}$ J. J. Hoyt, Acta Metall. Mater. 38, 1405-1412 (1990).

${ }^{46} \mathrm{D}$. DeFontaine, "A computer simulation of the evolution of coherent composition variations in solid solutions," Ph.D. thesis (Northwestern University, 1967).
${ }^{47}$ D. A. Cogswell, "A phase-field study of ternary multiphase microstructures," Ph.D. thesis (MIT, 2010).

${ }^{48}$ L.-Q. Chen, Acta metal. mater. 42, 3503-3513 (1994).

${ }^{49}$ C. Huang, M. Olvera de la Cruz, and B. W. Swift, Macromolecules 28, 7996-8005 (1995).

${ }^{50} \mathrm{C}$. Huang, M. Olvera de la Cruz, and P. W. Voorhees, Acta Mater. 47, 4449-4459 (1999).

${ }^{51}$ T. Philippe, D. Blavette, and P. W. Voorhees, J. Chem. Phys. 141, 124306 (2014). 